Preprint of : Gobet, F. (2002). Travailler avec Herbert Simon. Revue d'Intelligence Artificielle,16, 29-37.

\title{
Travailler avec Herbert A. Simon
}

\author{
Fernand Gobet
}

School of Psychology

University of Nottingham

Nottingham NG7 2RD

United Kingdom

frg@psyc.nott.ac.uk

RÉSUMÉ. Cet article décrit quelques aspects de ma collaboration avec Herbert Simon, qui dura plus de 10 ans, dont 6 à l'Université de Carnegie Mellon. Après avoir résumé les travaux empiriques et théoriques que nous avons effectués sur la mémoire des experts, il présente quelques aspects frappants de sa personnalité et décrit son style scientifique.

ABSTRACT. This article describes some aspects of my collaboration with Herbert Simon, which lasted over 10 years, including 6 at Carnegie Mellon University. After a summary of the empirical and theoretical work that we carried out on experts' memory, it documents some striking aspects of his personality and describes his scientific style.

MOTS-CLÉS: expertise, Herbert Simon, mémoire, modélisation par ordinateur, perception, recherche, reconnaissance de patterns, résolution de problème

KEY WORDS: computer modelling, expertise, Herbert Simon, pattern recognition, memory, perception, problem solving, search 


\section{Introduction}

Le trait le plus original d'Herbert Simon était sans doute sa capacité à appliquer une seule question (celle de la prise de décision) à de nombreux champs de connaissance, qu'il maîtrisait parfaitement. Peu possèdent sa capacité à dominer tant de domaines. Et bien que nous ayons abordé ensemble nombre de sujets lors de nos discussions, ma collaboration effective avec lui s'est essentiellement confinée à la psychologie. Ce récit va ainsi être biaisé dans la direction de mes intérêts scientifiques-le lecteur pardonnera cette rationalité (très) limitée. ${ }^{1}$

\section{Recherches sur la mémoire des experts}

En 1989, alors que je préparais ma thèse de doctorat, consacrée à la mémoire des joueurs d'échecs, j'obtins une bourse de recherche pour passer une année à l'Université de Carnegie Mellon (CMU) avec Simon. La lettre d'introduction que je lui envoyai était assez vague, et les seules qualifications que j'avançais étaient une licence en psychologie et un titre de maître international d'échecs. Simon, qui probablement y vit un moyen de réactiver ses travaux sur les échecs, sous couveuse depuis les années 70, m'invita.

Je vois encore notre première rencontre, par un magnifique matin de janvier. Sa place de travail chaleureuse, mais plutôt désorganisée, avec un bureau disparaissant sous des piles de livres et de documents. L'entretien fut bref-principalement une prise de contact—mais la cordialité de Simon me frappa d'emblée. Il me donna quelques conseils sur la vie à Pittsburgh et me décrivit brièvement ses recherches en cours.

Le deuxième entretien-le premier rendez-vous scientifique-m'apprit que, bien que Simon fût toujours ouvert à d'autres idées, il avait à cœur de développer avant tout les siennes. Mon projet contenait deux plans de recherche. Le premier, influencé par la technologie des systèmes experts, consistait à extraire les connaissances d'un maître d'échecs dans un domaine assez restreint (finale de tours avec quelques pions de chaque côté) et de programmer ces connaissances dans un modèle informatique. Un des intérêts du projet était de comparer la proportion de connaissances déclaratives et procédurales utilisées. Simon n'aima pas cette idée, qui lui semblait irréaliste ( «Un joueur comme Kasparov va vous donner un cours de plusieurs jours ; qu'allez-vous faire de toutes ces données ? »). Il indiqua clairement sa préférence pour

\footnotetext{
1 Le lecteur trouvera les références des travaux mentionnés dans ce texte dans l'article «Recherche et reconnaissance de patterns chez les experts » (ce numéro, pages $\mathbf{x x x}-\mathbf{y y y}$ ).
} 
un projet directement lié à la théorie du « chunking », cette théorie qu'il avait développée en compagnie de Chase dans les années 70 pour expliquer la mémoire remarquable des joueurs d'échecs. En particulier, il désirait réviser MAPP (Memory-Aided Pattern Perceiver), un programme informatique réalisant certains aspects de cette théorie.

Je dus donc me rabattre sur le second plan, moins élaboré, et passer une semaine plutôt angoissante à le raffiner. L'idée générale était de conduire plusieurs expériences sur la capacité de la mémoire des joueurs d'échecs et sur le degré de localisation spatiale de leurs connaissances. Avec du recul, il est évident que le deuxième thème correspondait beaucoup mieux à l'approche de Simon, avec un lien étroit entre modélisation et collecte de données expérimentales. De plus, réactiver MAPP signifiait utiliser EPAM (Elementary Perceiver and Memorizer), une théorie qui lui tenait à cœur mais qui n'avait pas eu l'impact espéré en psychologie.

Mon séjour à CMU, prévu pour une année, dura finalement six ans. Les raisons de cette prolongation sont simples : le privilège de collaborer avec Simon, ainsi que l'environnement interdisciplinaire et stimulant de CMU. Durant cette période, j'ai rencontré Simon en tête-à-tête presque hebdomadairement, en plus de séances de travail avec le groupe EPAM (voir plus bas). Nos entretiens suivaient en général l'ordre du jour que j'avais choisi, et se centraient sur notre recherche en cours; ils comprenaient également des discussions sur ses autres travaux de recherche, que ce soit en psychologie ou dans d'autres domaines.

Souvent, Simon commençait nos entretiens par un joyeux «Que savons-nous de nouveau sur les échecs aujourd'hui ?». Un de ses traits de caractère les plus frappants était sa curiosité immense et son appétit insatiable à découvrir de nouveaux phénomènes. Ses yeux brillaient chaque fois que j'amenais de nouvelles données expérimentales ou de nouvelles simulations, et il avait un plaisir évident-mais aussi une capacité déconcertante-à les analyser et à les comprendre. Typiquement, alors que ces données étaient devenues depuis longtemps inintéressantes pour moi car trop difficiles à comprendre, Simon me demandait d'effectuer de nouvelles analyses ou d'utiliser de meilleures représentations pour révéler les régularités cachées.

Bien que la plupart de nos rendez-vous aient été plutôt détendus, ils exigeaient également une concentration certaine. Il avait un style informel, assis sur une simple chaise, sans qu'un bureau ou une table ne s'interposât. J'oubliais parfois que je parlais à un des plus grands scientifiques du $20^{\text {ème }}$ siècle-jusqu'à ce que la réfutation tranchante de mon argument ou que la mention de son récent article en économie ou en philosophie me rappellât la vivacité 
fulgurante de son intelligence et mon ignorance dans la plupart des domaines qu'il maîtrisait. Bien qu'il eût une grande patience à écouter mes idées et arguments—et, je suppose, ceux d'autres étudiants et collaborateurs-il ne pouvait s'empêcher de contrecarrer ce qui lui semblait être une mauvaise idée. Il avait beaucoup d'élégance à le faire, mais il n'en restait pas moins que votre argument avait été, le plus souvent, irrémédiablement réfuté.

\subsection{Travaux empiriques}

Le point de départ de notre recherche fut donc la théorie du chunking et les difficultés empiriques qu'elle avait rencontrées. Les expériences de Chase et Simon, déjà, avaient produit un résultat inattendu : non seulement leur maître utilisait des chunks (groupes de pièces) relativement petits, mais il en replaçait davantage que l'amateur et la débutante qui servaient de contrôles. Or la théorie prédisait que les experts avaient les mêmes limites de mémoire à court terme (MCT) que les joueurs plus faibles. Cette anomalie, déjà notée dans l'article original, n'avait cessé de tourmenter Simon. En outre, plusieurs expériences sur les mnémonistes, que Chase et Ericsson avaient conduites dans les années 80, avaient démontré que les experts pouvaient encoder de l'information plus rapidement que prévu par la théorie. Comment pouvaient-ils le faire ? Simon pensait qu'ils utilisaient des «structures de retrait », c'est-à-dire des schémas en mémoire à long terme (MLT) leur permettant d'encoder rapidement de l'information. Mais qu'elle était la nature de ces structures ? Et comment les incorporer à MAPP ?

Sur le plan empirique, notre première ligne de recherche consista à développer une série d'expériences sur les joueurs d'échecs visant à explorer la capacité de leur MCT et la manière dont l'information est encodée en MLT. L'une de ces expériences répliqua l'étude de Chase et Simon avec davantage de sujets. Contrairement à l'étude originale, qui avait utilisé un échiquier et des pièces standard, nous eumes recours à une présentation par ordinateur, ce qui permit une collecte plus rapide et plus fiable des données. Les résultats établirent assez clairement que les maîtres utilisaient des chunks beaucoup plus grands que ce qui avait été trouvé dans l'étude originale, mais non pas plus nombreux que les joueurs plus faibles (en moyenne, tous les joueurs en utilisaient autour de 3). Simon avait depuis toujours eu un vif intérêt pour les paramètres de la cognition, et ce nombre lui semblait d'autant plus intéressant qu'il avait trouvé un résultat similaire dans son étude sur la mémoire d'idéogrammes chinois. 
Nous avons obtenu davantage de support pour ce paramètre lors d'une série d'expériences sondant les limites mnésiques des maîtres d'échecs. Dans ces expériences, nous présentions une séquence de positions durant quelques secondes chacune, et le joueur devait reconstituer l'ensemble des positions aussi précisément que possible. Une tâche très difficile, que seuls les maîtres arrivaient à surmonter. De manière intéressante, cependant, il semblait y avoir une limite autour de 3 ou 4 échiquiers-de nouveau le nombre magique !

La mémorisation de plusieurs échiquiers intrigua Simon, qui utilisa avec moi l'une de ses méthodes favorites : l'étude détaillée et longitudinale d'un seul sujet. Il me convainquit de commencer un entraînement consistant à augmenter le nombre de positions que je pouvais mémoriser. Durant près de deux ans, plusieurs fois par semaine, je m'asseyais donc en face de mon ordinateur et passais une demi-heure à mémoriser autant de positions qu'il était possible. L'expérience nous donna une quantité énorme de données, sur lesquelles nous étions encore en train de travailler, assez lentement, il est vrai, lors de son décès. Je passai de 4 échiquiers à un record de 10 échiquiers, mais ne pus jamais aller au-delà. Au début de nos entretiens, Simon s'enquerrait de mes progrès et de mes difficultés, et nous employions ces données pour essayer d'élaborer le concept de structure de retrait au sein de notre modèle.

D'autres expériences explorèrent l'effet de positions modifiées par image en miroir (qui nous donnèrent des informations utiles sur le type de représentation utilisé par les joueurs d'échecs), le rappel de positions aléatoires, et l'effet du temps de présentation. Cette dernière expérience confirma très nettement, avec une tâche spatiale, les paramètres temporels d'EPAM que Simon et Feigenbaum avaient estimés avec une tâche verbale (en particulier, à peu près 8 secondes pour créer une nouveau chunk). Mais c'est un autre résultat, que j'avais présenté plutôt comme une curiosité, qui piqua au vif l'intérêt de Simon. L'ex-champion du monde d'échecs Garry Kasparov avait défié plusieurs équipes nationales, affrontant en parties simultanées jusqu'à 8 maîtres ou grands-maîtres. Dans la plupart des cas, il avait remporté ces rencontres, mais, surtout, avait joué à un niveau qui le plaçait encore dans les cinq meilleurs joueurs du monde. Simon y vit immédiatement une illustration éclatante du rôle de la recherche heuristique et des connaissances dans la prise de décision.

\subsection{Modélisation : A la recherche des structures de retrait}

Un des aspects les plus gratifiants de notre collaboration était l'interaction serrée qu'il y avait entre la collecte des données et le développement d'un modèle. Les résultats empiriques 
contraignaient le modèle, et, à son tour, le modèle conduisait à de nouvelles expériences. La première version de CHREST (Chunk Hierarchy \& REtrieval STructures) amplifiait MAPP et le fusionnait avec PERCEIVER, un programme que Simon avait développé à la fin des années 60 pour simuler les mouvements oculaires des joueurs d'échecs. Une contribution intéressante fut d'imbriquer étroitement mécanismes de perception, d'apprentissage et de mémoire, et en particulier d'utiliser le concept de structure de retrait. Notre première hypothèse fut que les joueurs d'échecs utilisaient une structure de retrait assez similaire à celle qui avait été trouvée dans les recherches sur la mémoire des chiffres, c'est-à-dire une structure générique, utilisable avec n'importe quel matériel pour autant que l'on restât dans le domaine d'expertise. Avec une telle structure, CHREST répliqua plusieurs données expérimentales, mais avait également plusieurs faiblesses importantes : il surestimait la performance de rappel avec les positions aléatoires, et ne parvenait pas à simuler l'effet obtenu avec la modification par image en miroir. Cela nous amena à supposer, après beaucoup d'errements, que les structures de retrait étaient spécifiques, c'est-à-dire qu'elles étaient des schémas ne pouvant être reconnus que lorsque certaines configurations étaient présentes sur l'échiquier. Ce changement mena à des simulations nettement plus convaincantes. Un des intérêts de CHREST, bien au-delà de l'expertise aux échecs, est de contenir des mécanismes permettant de développer automatiquement des schémas, y compris les variables et les valeurs par défaut.

Durant la grande partie de mon séjour à CMU, le développement de CHREST alla de pair avec le développement d'EPAM-IV, qui modélisait la manière dont certains mnémonistes parviennent à mémoriser plus de 100 chiffres dictés à un rythme d'une seconde chacun. $\mathrm{Au}$ cours de rencontres plus ou moins hebdomadaires en compagnie d'Howard Richman, Jim Staszewski, et Shmuel Ur, nous discutions ces deux modèles, assez similaires puisqu'ils partagent la même architecture. Nous explorions également diverses applications d'EPAM : mémoire des experts, bien sûr, mais aussi formation de concepts, mémoire à court terme, et apprentissage verbal. Ces réunions étaient très vivantes et permettaient une portion nonnégligeable de brainstorming, et, bien que parfois un peu décousues, offraient un excellent environnement pour explorer l'architecture d'EPAM. Simon avait une présence non-directive, quoiqu'active.

\subsection{Publication d'articles}


La rédaction de la plupart des articles que nous avons écrits ensemble suivait un schéma assez standard. Je rédigeais un premier jet, centré sur la présentation des données empiriques, et l'article passait ensuite par une suite de révisions en commun. Il était fascinant de voir comment Simon parvenait à améliorer la qualité d'un document, avec parfois un minimum de changements. Comme d'autres visiteurs à l'anglais hésitant, j'ai pu apprécier la manière merveilleuse avec laquelle il enseignait les subtilités de sa langue ; sa patience à cet égard était remarquable. Son style avait une clarté et une simplicité d'expression hors du commun, qualités qu'il tenait à transmettre à ses coauteurs.

De manière surprenante, étant donné sa réputation, Simon eut passablement de difficultés à publier en psychologie durant la période 1991-1995. Cela était-il dû à son style scientifique très personnel ? Aux idées qu'il défendait—idées qui n’étaient pas toujours acceptées en psychologie ? Ou au fait qu'il soumettait un article pour publication relativement tôt, alors que d'autres auraient considéré quelque révision encore nécessaire (une application de sa théorie du « satisficing ») ? Il me confia un jour que, en fait, il ne comprenait pas la raison de cette « mauvaise passe ». Une autre surprise fut de voir sa réaction face aux rejets. On aurait pu penser que plus de 900 publications l'avaient rendu insensible ; au contraire, il était extrêmement mécontent et déçu, pestait contre l'ignorance des experts, et devait se forcer à oublier le rapport d'expertise durant quelques jours pour se calmer et prendre du recul.

Notre collaboration continua après mon départ pour Nottingham, principalement par émail, mais également par plusieurs courtes visites que j'effectuai à CMU. Une des plus mémorables fut celle que je fis peu après la victoire de Deeper Blue sur Gary Kasparov en 1997. Simon était évidemment très satisfait du résultat, qui d'une certaine manière confirmait sa prédiction de 1957 qu'un programme d'échecs allait devenir champion du monde dans les 10 ans (l'erreur de prédiction—40 ans plutôt que 10 ans—était plutôt petite, comme il me le fit remarquer). Toutefois, il y avait une certaine déception que le résultat ait été obtenu principalement par recherche brute, et non par recherche heuristique. De toute évidence, son intérêt pour les ordinateurs d'échecs provenait avant tout de son désir de comprendre les mécanismes de prise de décision chez les humains, et non pas du désir de développer un programme puissant par n'importe quel moyen.

\section{Simon tel que je l'ai connu}

Malgré son âge, Simon démontrait encore une énergie remarquable : il enseignait, faisait de la recherche, et participait à la vie politique de CMU. Quelle devait être son énergie quand il 
était plus jeune ? Il considérait le travail comme un hobby ( 80 heures par semaine lui semblait raisonnable) et il attendait de ses collaborateurs qu'ils partagent sa passion de la recherche. Le nombre d'heures passées à l'ouvrage était pour lui une condition incontournable pour être un scientifique performant—une application directe de ses travaux sur l'expertise montrant qu'il faut au moins 10.000 heures d'étude et de pratique pour devenir un expert. Le jeu en valait évidemment la chandelle, et, pour la majorité de ses collaborateurs et étudiants, les résultats faisaient plus que compenser le temps et l'énergie investis.

Comme je l'ai déjà mentionné, l'un des traits les plus frappants de Simon était sa curiosité insatiable. Il était intéressé à un nombre quasiment illimité de domaines, et, une fois qu'un problème ou une question s'était posé à lui, il n'avait de cesse jusqu'à ce qu'une solution ait été trouvée. Peu importe que la question fût importante ou non. Un signe que la recherche heuristique lui fournissait une satisfaction énorme. Cette attitude, parfois surprenante, dévoilait sans doute l'une de ses heuristiques de recherche les plus fécondes. Un autre aspect de cette curiosité était sa passion pour les langues. Dans la plupart des cas, il ne se faisait guère d'illusion sur son habileté à parler ces langues couramment, mais les maîtrisait assez pour lire des ouvrages complexes, y compris, pour le français, «A la recherche du temps perdu » de Proust, et ma thèse de doctorat !

Il était très généreux avec ses étudiants et collègues, et toujours prêt à nous soutenir dans les moments difficiles. Lorsque, pour quelque raison obscure, le département d'immigration américain me permit d'être employé par CMU, mais non d'être payé, Simon n'hésita pas à m'aider financièrement pendant quelques mois. Sa générosité s'étendait bien au delà du monde académique : il fit don aux œuvres de bienfaisance de l'argent associé à son prix Nobel.

Simon avait une méthode d'enseignement assez particulière. Il commençait ses classes en demandant s'il y avait des questions. Et il attendait que suffisamment de questions aient été posées. Trop peu de questions, pas de cours. Par la suite, il organisait son cours autour de ces questions, avec efficacité et virtuosité. Il était également un orateur remarquable, et prenait un plaisir certain à donner de nombreuses conférences sur le campus de CMU. Il avait gardé un style classique, narratif et concret, et n'employait pas de transparent—une gageure lorsque la discussion porte sur la représentation visuelle ou sur les algorithmes employés par EPAM ! Dans son enseignement comme dans ses conférences publiques, Simon avait également un don remarquable pour répondre aux questions les plus naïves avec respect, parvenant même à les tourner en questions intéressantes. 
Parfois, assez rarement il est vrai, il ne pouvait cacher une certaine amertume. Les sciences sociales utilisaient encore principalement des théories informelles, les économistes utilisaient encore des postulats d'optimalité intenables, en dépit de ses travaux sur la rationalité limitée, et la psychologie du traitement de l'information perdait de son influence face au connexionisme et aux neurosciences. Je me souviens d'une conférence donnée par Jay McClelland sur l'emploi de systèmes connexionnistes pour simuler des tâches de développement, où Simon intervint plutôt vertement pour montrer les limites de ces simulations et montrer qu'EPAM pourrait rendre compte de ces résultats de manière plus simple et plus élégante.

\section{Le style et les heuristiques scientifiques de Simon}

Il y avait un contraste dans le style scientifique de Simon. D'une part, ses recherches avaient une grande unité du fait de l'application systématique d'un thème principal (prise de décision chez les humains) à un grand nombre de domaines. D'autre part, sa curiosité indomptable et le plaisir évident qu'il avait à jouer avec des systèmes formels l'ont amené à effectuer des travaux portant sur toute une série de questions parfois disparates. Le contraste ne s'arrête pas là. Bien qu'il soit connu comme l'un des initiateurs de la révolution cognitiviste qui détrôna le behaviorisme, il était d'avis que la science est progressive et cumulative, et non pas révolutionnaire. Par exemple, les mécanismes d'apprentissage d'EPAM ont été directement influencés par un article écrit en 1940 par Eleanor Gibson, qui se situait alors dans la ligne officielle du behaviorisme.

Sur le plan empirique, Simon avait un penchant pour l'analyse fine des processus, employant des données empiriques aussi détaillées que possible, comme mouvements oculaires, types d'erreurs, temps de réaction, et, en particulier, protocoles verbaux. En général, il se sentait plus à l'aise dans l'analyse de plans expérimentaux simples, avec peu de sujets. Il avait coutume de dire que, en psychologie, les phénomènes intéressants sont si évidents qu'il n'est point nécessaire d'effectuer de test statistique-une hérésie dans un domaine scientifique si fier de sa sophistication méthodologique. Bien plus que de trouver des différences statistiquement significatives, son but était d'identifier, et de mesurer, les paramètres de la cognition humaine (par exemple, 8 secondes pour créer un chunk, ou 3 items pour la capacité de la MCT visuelle). Cette quête était motivée par son hypothèse que la cognition humaine est limitée et sérielle au niveau de l'attention. Ce désir de parcimonie théorique, on le retrouve dans ses travaux de modélisation, où il préférait des mécanismes simples et transparents-ce qui explique ses doutes pour des théories comme ACT-R, qui fusionne un nombre important de 
mécanismes, ou les systèmes connexionnistes, dont le comportement est difficile à comprendre. Avec ces mécanismes, son but était de développer des systèmes puissants, capable de comportements complexes et variés, mais avant tout de comportements semblables aux humains.

Il n'avait que peu de patience pour les théories formulées en termes vagues. Une des raisons pour laquelle il avait consacré sa carrière aux sciences sociales était d'y introduire plus de rigueur-d'abord avec les mathématiques, et ensuite avec la modélisation par ordinateur. Cela était toujours resté à l'ordre du jour, et il n'arrivait pas à cacher son agacement lorsqu'on lui parlait des arguments avancés par des philosophes comme Dreyfus ou Searle contre l'intelligence artificielle.

Au cours de nos entretiens, comme dans ses conférences et ses livres, Simon distillait toute une série de conseils sur la conduite de la recherche scientifique. En voici quelques-uns qui m'ont particulièrement marqué—bien que je ne les aie pas toujours suivis avec l'assiduité requise. D'abord, quelques conseils inspirés par sa théorie du satisficing. « Ce qui vaut la peine d'être fait vaut la peine d'être mal fait. Effectuez votre recherche avec soin, mais pas plus qu'il n'est nécessaire. » Le conseil suivant est très utile pour contrecarrer l'idée, plutôt paralysante pour de nombreux étudiants, qu'une thèse doit être une contribution majeure : «Une thèse n'est qu'un rapport de recherche. » D'autres conseils étaient liés à la manière de trouver le temps nécessaire pour maîtriser un domaine scientifique (et a fortiori plusieurs domaines) : «Votre temps est précieux. Ne le perdez pas à lire les journaux ou à regarder la télévision. Si quelque chose de vraiment important se passe dans le monde, vous le saurez par vos amis. » D'autres heuristiques avaient trait au contenu de la recherche : «Choisissez des questions importantes, mais aussi réalistes», et «Jouez avec vos connaissances, explorez des connexions inattendues. » Mais, de son avis même, l'heuristique qui l'avait le plus aidé était de s'entourer de nombreux collaborateurs, ou, plutôt, de nombreux amis. Je suis reconnaissant à Herbert Simon d'avoir utilisé cette heuristique, car elle m'a permis de rencontrer un scientifique hors pair, mais, surtout, un humaniste qui se souciait de ses semblables, et un ami.

Remerciements

Je tiens à remercier Jacques Pitrat et Bernard Plancherel pour leurs commentaires à propos de cet article. 